\title{
Multi-Group Maximum Entropy Model for Translational Non-Equilibrium
}

\author{
Vegnesh Jayaraman* \\ University of Illinois at Urbana-Champaign, Urbana, IL, USA \\ Yen $\mathrm{Liu}^{\dagger}$ \\ NASA Ames Research Center, Moffett Field, CA-94035, USA \\ Marco Panesi ${ }^{\ddagger}$ \\ University of Illinois at Urbana-Champaign, Urbana, IL, USA
}

\begin{abstract}
The aim of the current work is to describe a new model for flows in translational nonequilibrium. Starting from the statistical description of a gas proposed by Boltzmann, the model relies on a domain decomposition technique in velocity space. Using the maximum entropy principle, the logarithm of the distribution function in each velocity sub-domain (group) is expressed with a power series in molecular velocity. New governing equations are obtained using the method of weighted residuals by taking the velocity moments of the Boltzmann equation. The model is applied to a spatially homogeneous Boltzmann equation with a Bhatnagar-Gross-Krook ${ }^{1}$ (BGK) model collision operator and the relaxation of an initial non-equilibrium distribution to a Maxwellian is studied using the model. In addition, numerical results obtained using the model for a $1 \mathrm{D}$ shock tube problem are also reported.
\end{abstract}

\section{Introduction}

Reentry vehicles experience a wide range of flow environments as they travel through the earth's atmosphere, and the flow properties around the vehicle are influenced by a complex interaction of multiple physical processes. ${ }^{2}$ In particular, the presence of strong shocks and a rarefied flow regime result in non-equilibrium flow physics around the vehicle. The Navier Stokes equations, which are generally used to model continuum fluid flows, are derived from the Boltzmann equation ${ }^{3}$ under the assumption that the velocity distribution is a perturbed form of the equilibrium Maxwell-Boltzmann distribution. ${ }^{4}$ Such a fluid dynamic description is not appropriate for studying the flow physics around a reentry vehicle in the transitional and rarefied regimes. In these regimes, one of the best ways to describe flow processes is through the use of a statistical description based on kinetic theory of gases.

The Boltzmann equation is the governing equation describing the evolution of the distribution of molecular velocities. ${ }^{3,4}$ The distribution provides the number density of particles having a certain velocity at each point in physical space at a given time. The numerical solution of the Boltzmann equation involves discretization of three dimensions in velocity, three dimensions in space, and time. This process is computationally expensive and provides a description of the gas that is more detailed than required for most applications. One way to circumvent the problem is to use the method of weighted residuals to obtain macroscopic moment equations, thus reducing the number of degrees of freedom. $\mathrm{Grad}^{5}$ introduced moment methods for obtaining solutions to the Boltzmann equation. Levermore ${ }^{6}$ introduced a maximum entropy based closure for such methods. However, these methods allow for non physical solutions in certain cases. ${ }^{7,8}$ In addition, boundary conditions need to be specified for the higher order moments, like heat flux, which may

\footnotetext{
*Graduate Research Assistant, Department of Aerospace Engineering, University of Illinois at Urbana-Champaign, 302 Talbot Lab, 104 S. Wright St., Urbana, 61801, IL

${ }^{\dagger}$ Research Scientist, NASA Ames Research Center, Moffett Field, CA-94035, USA

$\ddagger^{\ddagger}$ Assistant Professor, Department of Aerospace Engineering, University of Illinois at Urbana-Champaign, 306 Talbot Lab, 104 S. Wright St., Urbana, 61801, IL
} 
not be easily feasible for a physical problem. In all of these methods, the entire velocity space is considered for obtaining the macroscopic model.

The approach proposed in this paper is to solve for the moments of the Boltzmann equation. The key difference between the previous moments based methods and the current method is that the solution is sought, in a piecewise manner, over a limited range of the velocity space. The velocity domain is decomposed into small groups, and the microscopic velocity distribution function in each of these groups is reconstructed using functions generated using the maximum entropy principle. The macroscopic governing equations are obtained by using the method of weighted residuals. A multi-group maximum entropy model has been derived earlier for thermo-chemical non-equilibrium. ${ }^{7,9}$ This paper shows the same methodology applied to translational non-equilibrium. Some similar approaches to the solution of kinetic equations are mentioned. A hybrid-kinetic approach has been formulated that assumes a Maxwellian distribution in a certain region of velocity space and solves the kinetic equation in the rest of the velocity space. ${ }^{10}$ A model for the specific case of two groups has been developed using methods similar to the one suggested in this paper. ${ }^{11}$ To the best of our knowledge, this is the first attempt to implement such a model for an arbitrary number of groups.

The paper is organized as follows. Section II briefly describes the microscopic governing equation and the BGK collision term and elaborates upon the sequence of steps used in the model derivation. Section III presents the results obtained when the model is applied to specific zero and one dimensional cases. Some concluding remarks are provided in Section IV. In Appendix, some of the mathematical expressions required for model solution are given.

\section{Theory}

This section is broadly divided into three major parts. The first part gives a brief overview of the Boltzmann Equation and the collision operator used for the current problem. The second part explains the maximum entropy methodology used for obtaining the group distribution functions. In the final part, the model governing equations are presented.

\section{II.A. Boltzmann Equation}

The Boltzmann equation describes the rate of the change of the distribution function of the gas molecules as a function of position, velocity, and time. This allows one to obtain a kinetic theory based description of various phenomena. The Boltzmann equation for a monoatomic gas is expressed $\operatorname{as}^{12}$ :

$$
\left(\frac{\partial}{\partial t}+\vec{c} \cdot \frac{\partial}{\partial \vec{x}}\right) f(\vec{x}, t ; \vec{c})=\mathcal{G}(f)-f \mathcal{L}(f)
$$

where $f$ is the distribution function, $\vec{x}$ is position space and $\vec{c}$ is velocity space. $\mathcal{G}(f)$ and $\mathcal{L}(f)$ are the nonlinear collision integral operators and represent the replenishing and depleting collision processes respectively. Equation (1) assumes that there are no external forces acting on the molecules. This equation is used as a starting point to derive the macroscopic governing equations of the current model.

\section{II.B. BGK model}

The non-linear collision terms in Eq.(1), which can be derived from knowledge of the intermolecular force field, pose a great challenge when one tries to solve the Boltzmann equation. The Bhatnagar-Gross-Krook ${ }^{1}$ (BGK) model provides a simplified approximation to the scattering process. The collision terms, based on the BGK model, can be expressed as ${ }^{12}$ :

$$
\begin{gathered}
\mathcal{L}(f)=\nu \quad \mathcal{G}(f)=\nu F_{m} \\
F_{m}=n\left(\frac{\beta}{\pi}\right)^{\frac{3}{2}} \exp \left(-\beta(\vec{c}-\vec{u})^{2}\right)
\end{gathered}
$$

where $n, u$ and $T=\frac{1}{2 R \beta}$ are the local macroscopic number density, velocity, and temperature respectively, $F_{m}$ is the Maxwellian Distribution and $R$ is the gas constant. The variable $\nu$ is a collision frequency that typically depends on the state of the gas and the number density but not on the molecular velocity. ${ }^{3}$ The 
local density, velocity and temperature are obtained by taking the velocity moments of the distribution function:

$$
\begin{aligned}
n & =\int f d \vec{c} \\
n \vec{u} & =\int \vec{c} f d \vec{c} \\
2 R T=\frac{1}{\beta} & =\frac{2}{3 n} \int m(\vec{c}-\vec{u})^{2} f d \vec{c}
\end{aligned}
$$

where $m$ is the mass of the molecule.

The depleting collisions are represented by the $-\nu f$ term, and the replenishing collisions are represented by $\nu F_{M}$. The form of the replenishing collisions suggests that the molecules are undergoing a relaxation process to a Maxwellian distribution at the local mean velocity, density and temperature. These simplifications are inspired for the case of Maxwell molecules and rigid spheres ${ }^{3,12}$ and further details can be obtained in the references.

The Krook equation, obtained by replacing the collision term in equation Eq.(1) with the BGK model terms, is given by:

$$
\left(\frac{\partial f}{\partial t}+\vec{c} \cdot \frac{\partial f}{\partial \vec{x}}\right)=\nu\left(F_{m}-f\right)
$$

The Krook Equation is still a non-linear equation, but the collision term is simpler when compared to the Boltzmann Equation.

\section{II.C. Group Distribution Function}

It is known from thermodynamic principles that the entropy of a system is maximized when it reaches an equilibrium state. This property is used in statistical mechanics and kinetic theory to obtain the energy and velocity distribution of particles at a particular temperature, respectively. The collision integral for a bimolecular collision in Boltzmann equation is given $\operatorname{as}^{3}$ :

$$
\left[\frac{\partial}{\partial t}(f)\right]_{\text {coll }}=\int_{-\infty}^{\infty} \int_{0}^{4 \pi}\left[f\left(c_{i}^{\prime}\right) f\left(z_{i}^{\prime}\right)-f\left(c_{i}\right) f\left(z_{i}\right)\right] g \sigma d \Omega d V_{z}
$$

A necessary and sufficient condition for equilibrium is given by: ${ }^{3}$

$$
\begin{aligned}
f\left(c_{i}^{\prime}\right) f\left(z_{i}^{\prime}\right) & =f\left(c_{i}\right) f\left(z_{i}\right) \\
\ln \left(f\left(c_{i}^{\prime}\right)\right)+\ln \left(f\left(z_{i}^{\prime}\right)\right) & =\ln \left(f\left(c_{i}\right)\right)+\ln \left(f\left(z_{i}\right)\right)
\end{aligned}
$$

From Eq.(10), it is observed that $\ln (f)$ is a collisional invariant. Hence, $\ln (f)$ is expressed as linear combination of the collisional invariants from classical mechanics:

$$
\ln (f(\vec{c}))=\alpha+\vec{\gamma} \cdot \vec{c}+\delta \vec{c} . \vec{c}
$$

The maximum entropy principle has been used in information theory, ${ }^{13,14}$ as interpolants, ${ }^{15}$ and as a method for providing solutions to under-determined inverse problems. ${ }^{16}$

The mathematical formulation for obtaining the group distribution function can be stated as: Obtain a function $f_{k}$ such that entropy of this function is maximized and the first 3 velocity moments of $f_{k}$ are equal to a given value of the moments $\left(\mu_{n, k}\right)$ where $n=0,1,2$ corresponds to the order of the moment. The maximum entropy functional can be defined as:

$$
\mathcal{J}\left[f_{k}\right]=\int_{\vec{c}_{k}}^{\vec{c}_{k+1}}\left(-f_{k} \ln f_{k}+f_{k}+\alpha_{k} f_{k}+\vec{\gamma}_{k} \cdot \vec{c} f_{k}+\delta_{k} c^{2} f_{k}\right) d \vec{c}-\alpha_{k} \mu_{0, k}-\vec{\gamma}_{k} \cdot \vec{\mu}_{1, k}-\delta \mu_{2, k}
$$

where $\alpha_{k}, \vec{\gamma}_{k}$ and $\delta_{k}$ are the lagrange multipliers. $\vec{c}$ is the velocity vector corresponding to the three dimensions in velocity space. The functional variation of equation (12) results in:

$$
\ln \left(f_{k}\right)=\alpha_{k}+\vec{\gamma}_{k} \cdot \vec{c}+\delta_{k} \vec{c} \cdot \vec{c}
$$


In the current case, the velocity space is divided into multiple groups and a maximum entropy based function representation is assumed for the distribution function within each group. The expression for the distribution function is given by:

$$
\begin{gathered}
f(\vec{x}, t ; \vec{c})=\sum_{k=1}^{k=N_{g}} f_{k}(\vec{x}, t ; \vec{c}) \\
f_{k}(\vec{x}, t ; \vec{c})= \begin{cases}A_{k} \exp \left(-\beta_{k}\left(\vec{c}-\vec{w}_{k}\right)^{2}\right), & \text { if } \vec{c}_{k} \leq \vec{c} \leq \vec{c}_{k+1} \\
0, & \text { otherwise }\end{cases}
\end{gathered}
$$

where $k$ is the group index, $N_{g}$ is the number of velocity groups, $c_{k}$ and $c_{k+1}$ are the $k^{\text {th }}$ group's lower and upper velocity bounds and $A_{k}, \beta_{k}$ and $w_{k}$ are the function parameters. The spatial and temporal dependence of the distribution function appears in the number density and the function parameters. The expressions in equations (13) and (15) can be shown to be equivalent.

The expression given in Eq.(15) is used as an approximation of the velocity distribution function of each velocity group. By using such an approximation, the particles with velocities in a specific group are assumed to be present in a local equilibrium with each other, but in non-equilibrium with the particles in other groups. The schematic for model formulation, illustrating the velocity groups and the distribution function within each group, is shown in Figure 1.

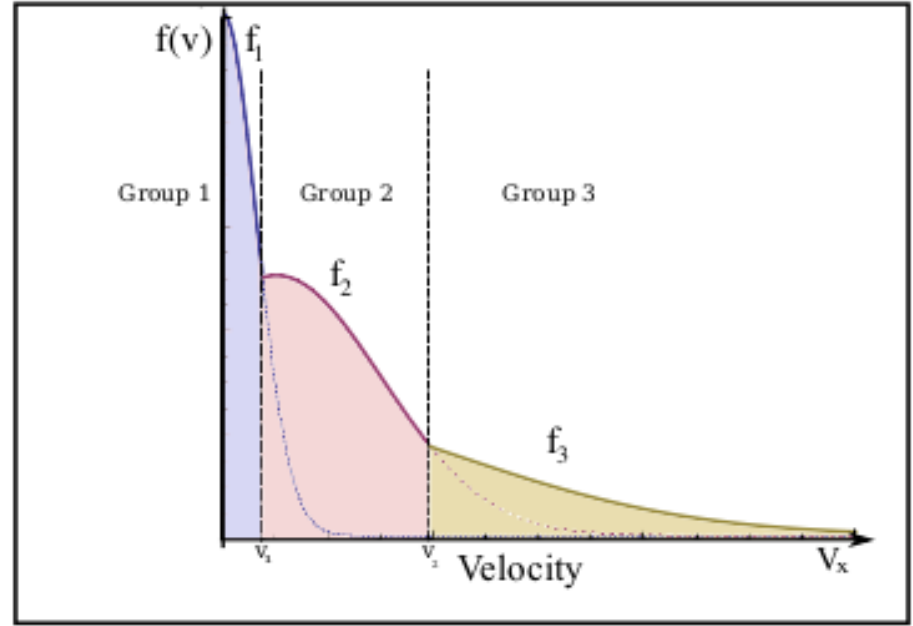

Figure 1: Piecewise representation of velocity distribution function using 3 groups.

\section{II.D. Model Governing Equations}

The velocity moments of the Krook Equation are taken to obtain the macroscopic governing equations for each group. All the cases discussed in this paper consider either zero or one dimension in space and 3 dimensions in velocity. No external forces are assumed to act on the system. The Krook equation with these assumptions, becomes:

$$
\frac{\partial f}{\partial t}+c_{x} \frac{\partial f}{\partial x}=\nu\left(F_{m}-f\right)
$$

In all the test cases considered, mean flow is assumed only along the $x$ direction. Along $y$ and $z$ direction, the profile is considered to be a Maxwellian at the instantaneous local temperature, with zero mean flow. The group distribution function that is taken for all cases is given by:

$$
f_{k}(x, t ; \vec{c})=A_{k} \exp \left(-\beta_{k}\left(\left(c_{x}-w_{k}\right)^{2}+c_{y}^{2}+c_{z}^{2}\right)\right), \quad \forall c_{y}, c_{z}, c_{x, k} \leq c_{x} \leq c_{x, k+1}
$$


The macroscopic governing equations are obtained by taking velocity moments:

$$
\begin{array}{r}
\int_{c_{x}=c_{x, k}}^{c_{x, k+1}} \int_{c_{y}=-\infty}^{\infty} \int_{c_{z}=-\infty}^{\infty} m\left(\frac{\partial f_{k}}{\partial t}+c_{x} \frac{\partial f_{k}}{\partial x}=\nu\left(F_{m}-f_{k}\right)\right) d c_{x} d c_{y} d c_{z} \\
\int_{c_{x}=c_{x, k}}^{c_{x, k+1}} \int_{c_{y}=-\infty}^{\infty} \int_{c_{x}=c_{x, k}}^{\infty} m c_{x}\left(\frac{\partial f_{k}}{\partial t}+c_{x} \frac{\partial f_{k}}{\partial x}=\nu\left(F_{m}-f_{k}\right)\right) d c_{x} d c_{y} d c_{z} \\
\int_{c_{z}=-\infty}^{\infty} m\left(c_{x}^{2}+c_{y}^{2}+c_{z}^{2}\right)\left(\frac{\partial f_{k}}{\partial t}+c_{x} \frac{\partial f_{k}}{\partial x}=\nu\left(F_{m}-f_{k}\right)\right) d c_{x} d c_{y} d c_{z}
\end{array}
$$

These can then be written as:

$$
\frac{\partial \vec{U}_{k}}{\partial t}+\frac{\partial \vec{F}_{k}}{\partial x}=\nu\left(\vec{U}_{k}^{M}-\vec{U}_{k}\right)
$$

where $\vec{U}_{k}$ is expressed as :

$$
\vec{U}_{k}=\left[\begin{array}{c}
\rho_{k} \\
\rho_{k} u_{k} \\
\rho_{k} e_{k}
\end{array}\right]=\int_{c_{x}=c_{x, k}}^{c_{x, k}} \int_{c_{y}=-\infty}^{\infty} \int_{c_{z}=-\infty}^{\infty}\left(\begin{array}{c}
m \\
m c_{x} \\
m\left(c_{x}^{2}+c_{y}^{2}+c_{z}^{2}\right)
\end{array}\right) f_{k} d c_{x} d c_{y} d c_{z}
$$

and $\vec{F}_{k}$ can be obtained by:

$$
\vec{F}_{k}=\int_{c_{x}=c_{k, x}}^{c_{k+1, x}} \int_{c_{y}=-\infty}^{\infty} \int_{c_{z}=-\infty}^{\infty}\left(\begin{array}{c}
m c_{x} \\
m c_{x}^{2} \\
m\left(c_{x}^{2}+c_{y}^{2}+c_{z}^{2}\right) c_{x}
\end{array}\right) f_{k} d c_{x} d c_{y} d c_{z}
$$

$\rho_{k}, \rho_{k} u_{k, x}$ and $\rho_{k} e_{k}$ are the density, momentum and energy contribution from group $k$ respectively. $\vec{F}_{k}$ is the flux vector. The detailed expressions for $\vec{F}_{k}$ and $\vec{U}_{k}$ in terms of group function parameters are given in Appendix. $\vec{U}_{k}^{M}$ corresponds to the the value of $\vec{U}_{k}$ if $\beta_{k}, w_{k}$ and $A_{k}$ are replaced by the corresponding local Maxwellian distribution's values. $\vec{U}_{k}^{M}$ can be viewed as the fraction of the equilibrium density, momentum, and energy that is present is the $k^{t h}$ velocity group.

\section{Results}

Two different cases are studied in this paper. The first case involves the relaxation of a spatially homogeneous system . In the second case, the 1D Sod-Shock Tube problem is solved.

\section{III.A. Case 1: Zero Dimensional (Space) Relaxation}

A spatially homogeneous system is chosen as a simple test case for testing the accuracy of the macroscopic grouping model and to test the inversion routines used to obtained the group function parameters from the macroscopic variables. The Krook equation Eq.(16) simplifies to :

$$
\frac{\partial f}{\partial t}=\nu\left(F_{m}-f\right)
$$

Equation Eq.(22) has an analytical solution when the collision frequency $(\nu)$ is constant and the system is a closed system. The macroscopic governing equations become:

$$
\begin{aligned}
\frac{\partial \rho_{k}}{\partial t} & =\nu\left(\rho_{k}^{M}-\rho_{k}\right) \\
\frac{\partial \rho_{k} u_{k}}{\partial t} & =\nu\left(\rho_{k}^{M} u_{k}^{M}-\rho_{k} u_{k}\right) \\
\frac{\partial \rho_{k} e_{k}}{\partial t} & =\nu\left(\rho_{k}^{M} e_{k}^{M}-\rho_{k} e_{k}\right)
\end{aligned}
$$




\section{III.A.1. Case 1: Initial Condition}

The initial condition chosen for case 1 is a bimodal distribution in x-velocity space centered about $c_{x}=0$. Such a bimodal distribution can be expressed as:

$$
f_{i}(\vec{c})=\frac{n}{2}\left(\frac{b}{\pi}\right)^{1.5}\left(\exp \left(-b\left(c_{x}-v\right)^{2}\right)+\exp \left(-b\left(c_{x}+v\right)^{2}\right)\right) \exp \left(-b c_{y}^{2}\right) \exp \left(-b c_{z}^{2}\right)
$$

For a closed system, in the absence of energy addition, Eq.(26) relaxes to a Maxwellian distribution of the form:

$$
F_{m}=n\left(\frac{b_{m}}{\pi}\right)^{1.5} \exp \left(-b_{m}\left(c_{x}^{2}+c_{y}^{2}+c_{z}^{2}\right)\right)
$$

where

$$
\frac{3}{2 b_{m}}=\frac{3}{2 b}+v^{2}
$$

For the test problem, velocity variables are non-dimensionalized by the mean speed of the Maxwellian and time is non-dimensionalized by the reciprocal of collision frequency $\nu$.

The initial non-dimensionalized non-equilibrium distribution is chosen to be a bimodal distribution of the form:

$$
f_{i}(\vec{c})=\sqrt{\frac{3}{4 \pi}}\left(\exp \left(-3\left(c_{x}-\frac{1}{\sqrt{3}}\right)^{2}\right)+\exp \left(-3\left(c_{x}+\frac{1}{\sqrt{3}}\right)^{2}\right)\right) \times \frac{3}{\pi} \exp \left(-3\left(c_{y}^{2}+c_{z}^{2}\right)\right)
$$

The corresponding Maxwellian distribution that the above non-equilibrium relaxes to is given by:

$$
f_{M}(\vec{c})=\frac{1}{\pi^{\frac{3}{2}}} \exp \left(-\left(c_{x}^{2}+c_{y}^{2}+c_{z}^{2}\right)\right)
$$

The initial distribution along the $\mathrm{x}$-direction and the final maxwellian distribution that the initial distribution relaxes to are plotted in Figure 2. A structureless gas is chosen and the model accounts for three dimensions in velocity space. It is assumed that the current case corresponds to an isolated system with no addition of particles or energy into the system.

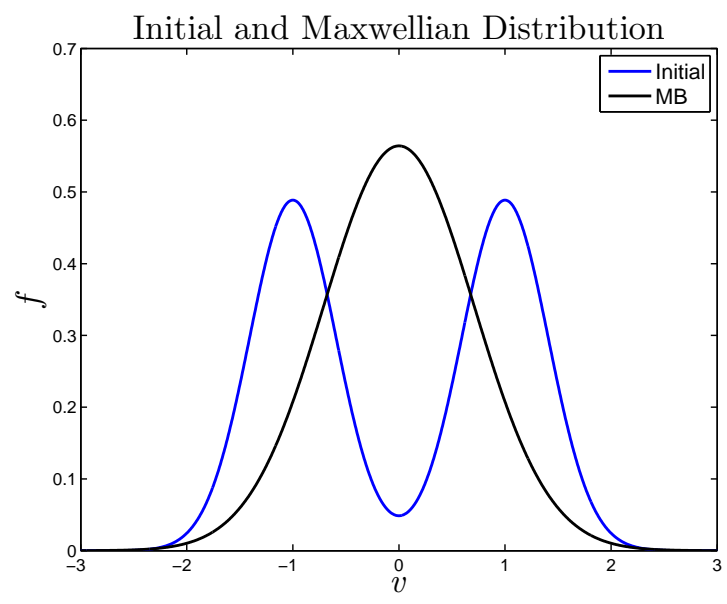

Figure 2: Initial distribution and corresponding Maxwell Boltzmann Distribution.

\section{III.A.2. Inversion}

In order to obtain the instantaneous distributions in the zero dimensional test case and the value of the flux variables in the one dimensional test case, the group function parameters need to be obtained from the moment values. The expressions for the moment values, given in Appendix, as functions of the group parameters is nonlinear in nature. Specifically, referring to Eq.(17), the expressions are linear in the parameter $A_{k}$ 
and nonlinear in $\beta_{k}, w_{k}$. The group velocity and group energy moment values are normalized with respect to the group density moment to eliminate $A_{k}$, and two nonlinear equations are solved for the $\beta_{k}$ and $w_{k}$.

The Newton-Raphson algorithm was used for finding the roots of the nonlinear equations. One major issue faced during inversion is that the group density value could be arbitrarily low, causing roundoff errors to corrupt the normalization. This prevents the Newton-Raphson algorithm from converging to a solution. To overcome this problem, a large table was generated to obtain $\beta_{k}$ and $w_{k}$ given the normalized group energy and group velocity. The surface plot showing $\beta_{k}$ and $w_{k}$ is shown in Figure 3. From the plots, it is observed that there is a clustering of points at specific edges of the surface (signified by the clustering of lines at the left and right edges of Figure $3 \mathrm{a}$ and the left edge of Figure 3b). At these edge locations, the group density is extremely low. To get the solution at these locations, the surfaces in Figure 3 are interpolated, and the interpolated values are used for distribution function reconstruction or flux evaluation.

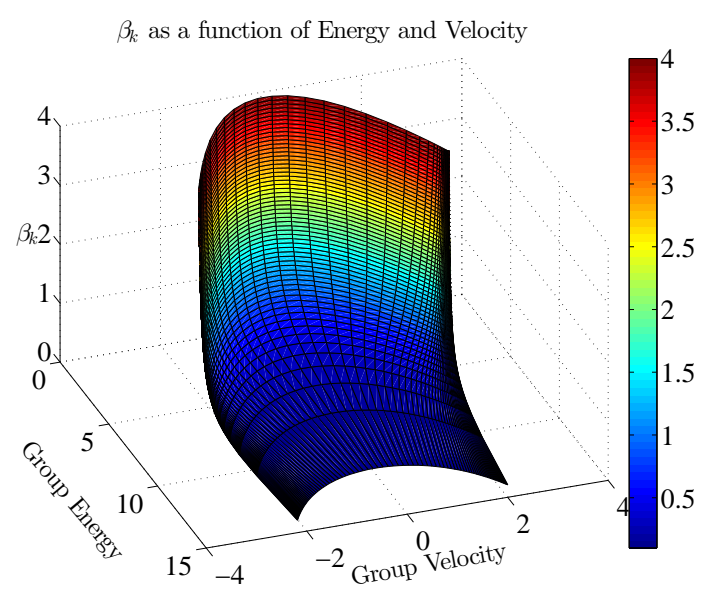

(a) $\beta_{k}$ surface plot

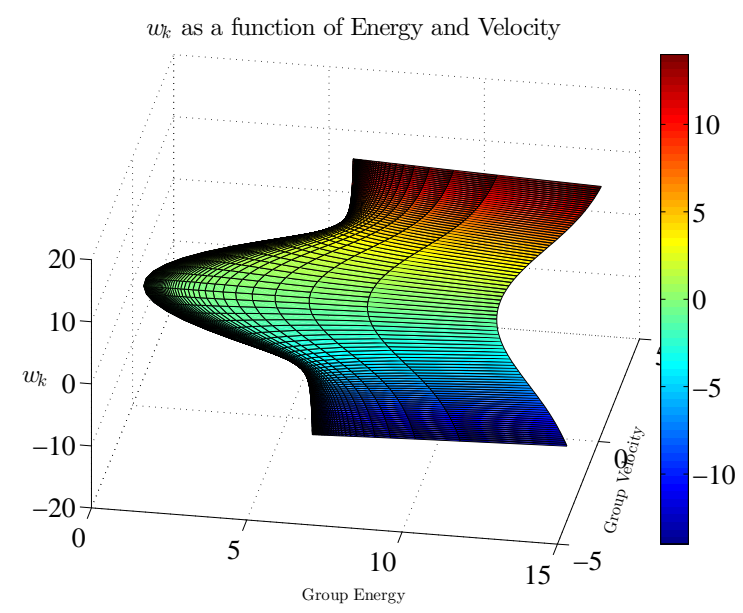

(b) $w_{k}$ surface plot

Figure 3: Inversion Surface Plots,$\beta_{k}$ or $w_{k}$ as function of normalized group energy and velocity

\section{III.A.3. Case 1: Results}

Three macroscopic governing equations, (Eqs.(23)-(25)) for group density, momentum, and energy are solved to obtain the variation of each macroscopic moment as a function of time. The velocity distribution function is symmetric about the origin. Hence, the numerical solution is plotted only in the positive half of the $\mathrm{x}$-velocity space. The solution in the other is a mirror image of the solution in the positive half about the y-axis.

The parameters used for obtaining the Boltzmann equation solution and the macroscopic model solutions are :

$$
\begin{aligned}
N_{g} & =13 \\
\nu & =1
\end{aligned}
$$

$N_{g}$ is the number of groups the velocity space is divided into. The relaxation process is run from nondimensional time $t=0$ to $t=50$. The velocity space between $0 \leq v \leq 3$ is divided into 12 equally spaced groups and the velocity space from $3<v<\infty$ is modeled as a single group. This is because the value of the distribution function is of the order machine precision for high velocities and causes numerical errors if more than one group is used in this region.

The numerical solution of the multi-group maximum entropy model and the Boltzmann equation with the BGK collision operator are plotted at different times in Figs. 4b and 4a respectively. In order to compare 


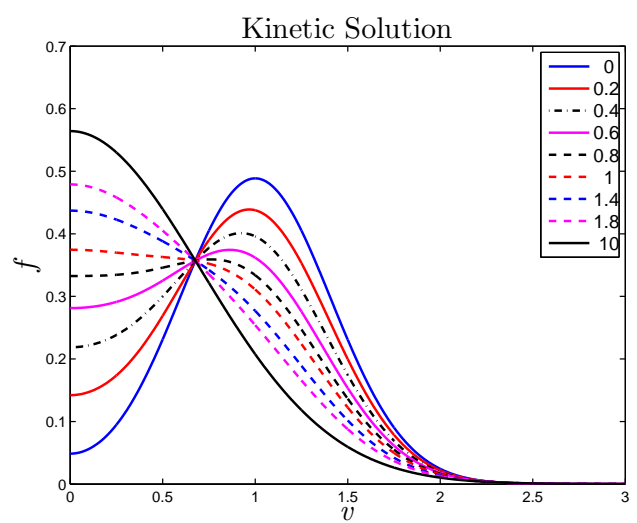

(a) Kinetic Solution

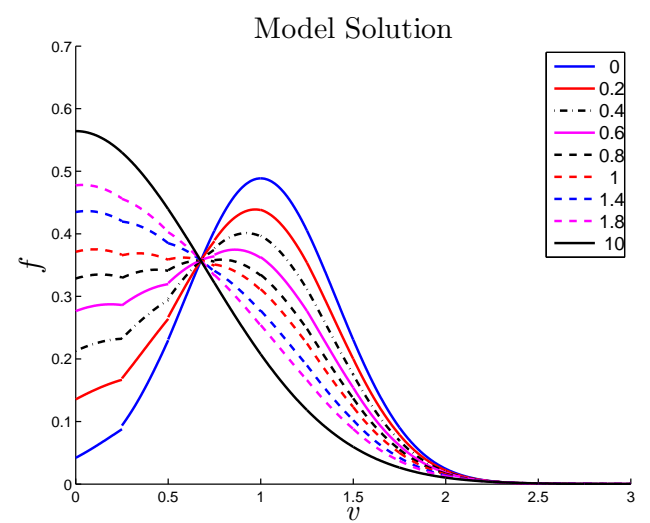

(b) Model Solution

Figure 4: 0D Relaxation Solution at different times. Kinetic (4a) and Model(4b) solution

Solution Comparison, time $=0$

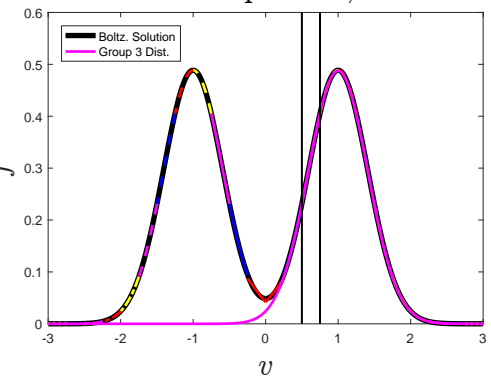

Solution Comparison, time $=0.6$

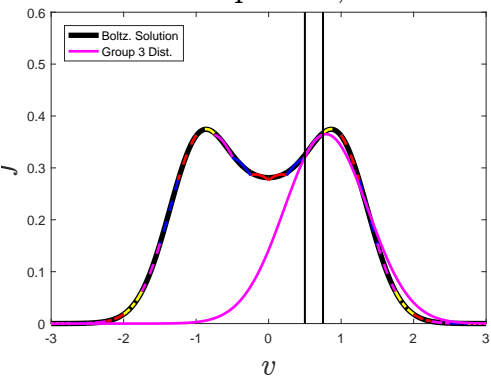

Solution Comparison, time $=1.4$

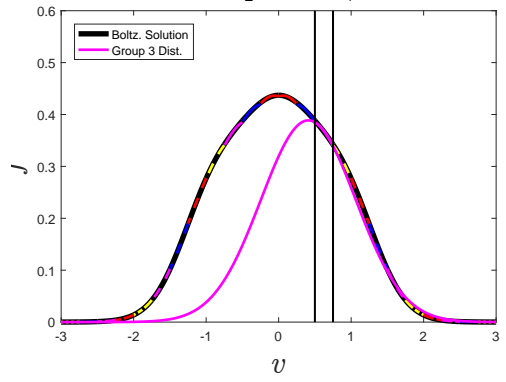

Solution Comparison, time $=0.2$

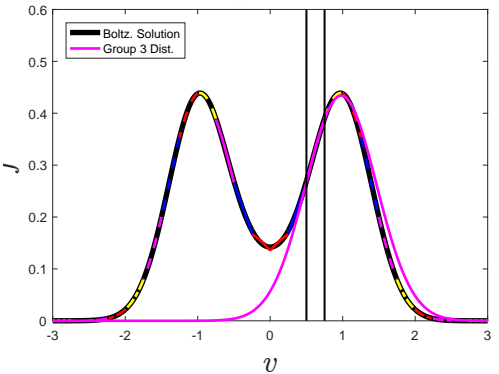

Solution Comparison, time $=0.8$

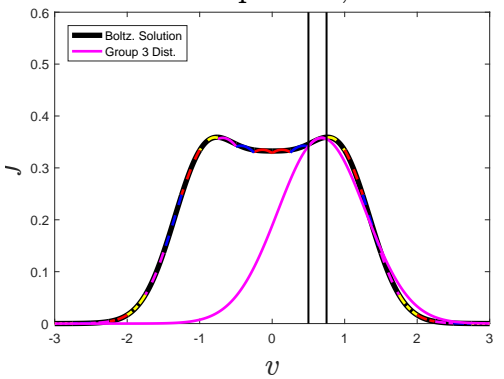

Solution Comparison, time $=1.8$

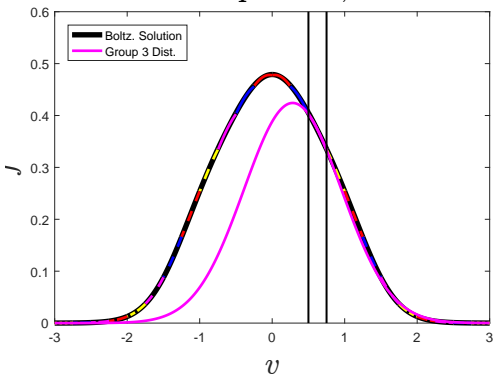

Solution Comparison, time $=0.4$

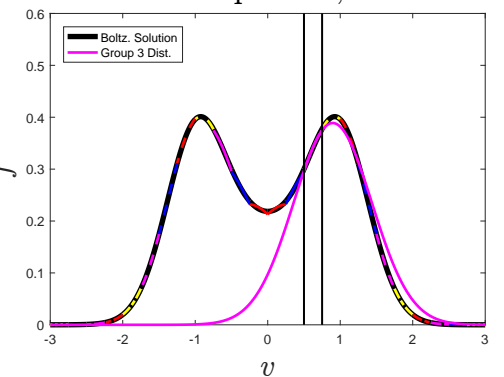

Solution Comparison, time $=1$

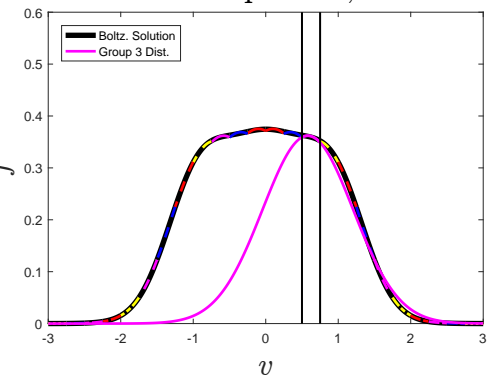

Solution Comparison, time $=10$

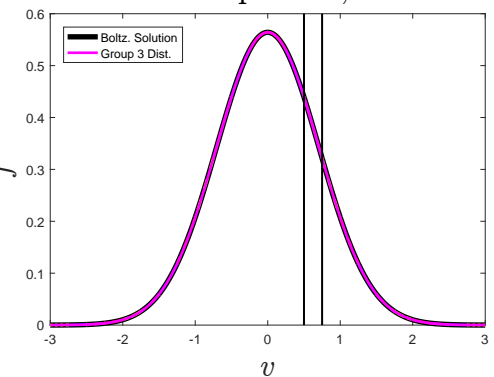

Figure 5: Comparison Plot - Kinetic Solution, Model Solution and Group Solution 
the solutions of the macroscopic model with the exact solution, the model solution is plotted overlapping with the corresponding Boltzmann equation solution in Figure. 5 for 10 different times. The model solution appears to closely agree with the exact solution. In addition, solution corresponding to a particular group is plotted over the entire velocity space. The group velocity parameter value need not lie within the group velocity bounds. As time progresses, the group velocity goes from a positive value to 0 and the group temperature increases as expected. The final group solution and the Maxwellian at time $t=10$ is identical. The primary advantage of the model lies in the fact that a solution using 13 groups requires us to solve 39 ordinary differential equations (ODE), while the exact solution requires us to solve 10,000 ODE's ( at each point in the discretized velocity space). This test case was performed to test the ability of the model to capture the microscopic distributions and the inversion methodology.

\section{III.B. Case 2 : One Dimensional Flow - Shock Tube}

In this section, the model equations (Eq. 21) are used to solve a shock tube problem. The physical domain is $0 \leq x \leq 1$. The initial conditions chosen are: ${ }^{17}$

$$
\begin{array}{rl}
\rho_{L}=1 & \rho_{R}=0.125 \\
u_{L}=0 & u_{R}=0 \\
P_{L}=1 & P_{R}=0.1 \\
0 \leq x \leq 0.5 & 0.5<x \leq 1
\end{array}
$$

where $\rho$ is the density, $P$ is the pressure, $u$ is the mean flow velocity, and $L, R$ denote the left and right half of the domain respectively. The gas parameters are non-dimensionalized by the upstream conditions, the velocity is non dimensionalized by the mean speed. Time is non dimensionalized using ratio of the length of the domain and the mean speed. The distribution function at each grid point is assumed Maxwellian at $t=0$. The Maxwellian at each physical location can be represented by Eq. (3). The corresponding distribution function parameters expressed in terms of the initial conditions are:

$$
\begin{aligned}
n_{L}=\rho_{L} & n_{R}=\rho_{R} \\
\beta_{L}=\frac{\rho_{L}}{2 P_{L}} & \beta_{R}=\frac{\rho_{R}}{2 P_{R}} \\
u_{L}=0 & u_{R}=0
\end{aligned}
$$

The governing equations are integrated in time using Runge Kutta fourth order time stepping method(RK4). The spatial derivatives are obtained using a 2nd order central difference scheme or characteristics based upwinding scheme. The latter scheme was developed in order to exploit the hyperbolic nature of the system of equations but is computationally more expensive than the central scheme implementation. At the left and right boundaries, one sided stencils are used for obtaining derivatives. The test gas is assumed to have $\gamma=\frac{5}{3}$.

The model solution is compared with the corresponding kinetic solution. The kinetic solution is obtained by solving the boltzmann equation. The specific solver used for the kinetic solution uses discontinuous galerkin based discretizations in physical and velocity space. ${ }^{18,19}$

\section{III.B.1. High Collision Frequency Results}

In this study, 3 groups in velocity space $([\infty,-1],[-1,1]$ and $[1, \infty])$ are used. The velocity bounds specified are the non-dimensionalized values. Physical space is discretized using 400 grid points. The model results reported use 2 nd central difference for spatial derivatives. The time step for RK4 is taken to be $10^{-5}$. The simulation is run till a non dimensional time of $t=0.2$. For this time, the flow features have not reached the wall and the boundary conditions do not affect the flow solution.

The model solution was compared with the kinetic solution for two different tests - a high collision frequency $(\nu=10000) /$ low knudsen number $(\nu \propto 1 / K n)$ test case (Euler), a low collision frequency $(\nu=100)$ case (near end of continuum / or beginning of transition regime). Figure 6 shows the model results for the high collision frequency $(\nu=10000)$ case. This case can be interpreted as a limiting case corresponding to an Euler solution. The high collision frequency value corresponds to the molecules relaxing to the equilibrium distribution rapidly. The model solution is plotted alongside the numerical solution to 

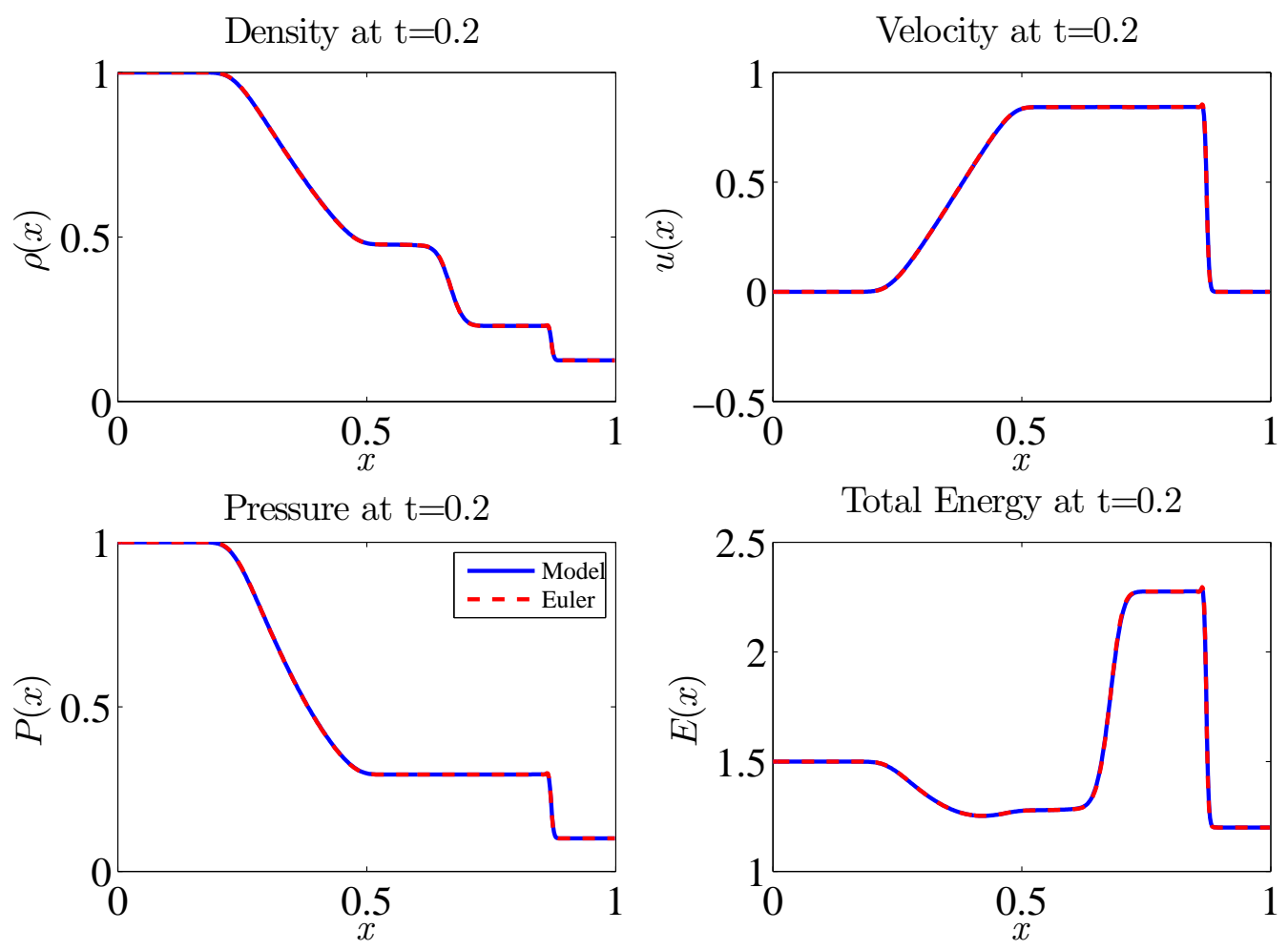

Figure 6: Sod shock tube problem solution, $\mathrm{t}=0.2 \mathrm{sec}$, Collision Frequency $\nu=10000$
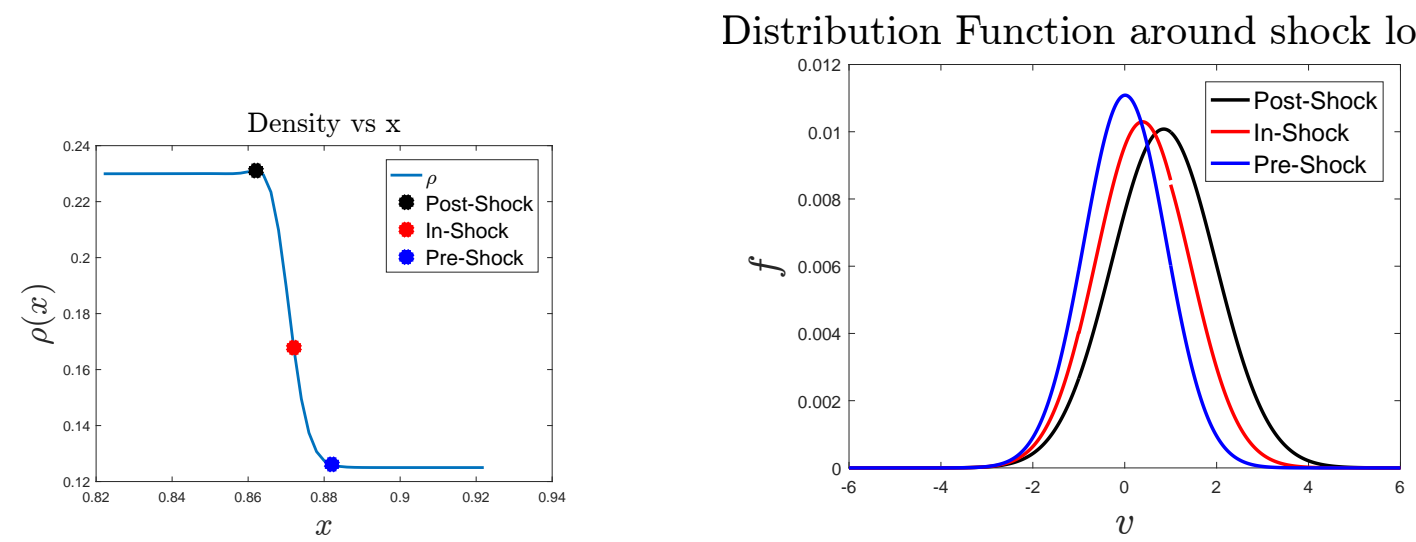

(a) Shock Schematic

(b) Distribution Function

Figure 7: Distribution Function in the Shock Region, $\nu=10000$

the Euler equations for the same set of initial conditions in Figure 6. The model results overlap the Euler solutions to graphic accuracy.

The distribution function within the shock is plotted for $\nu=10000$ (Figure 7b). For the high collision frequency case, the model solution predicts that the distribution function goes from one Maxwellian to 
another. This is expected as the Euler equation model assumes translational equilibrium.

\section{III.B.2. Low Collision Frequency Results}

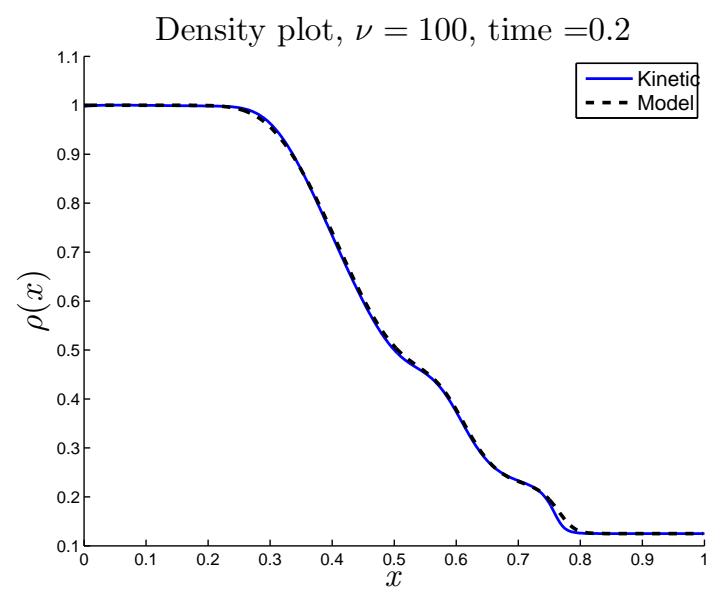

(a) Shock Tube , $\nu=100$ case

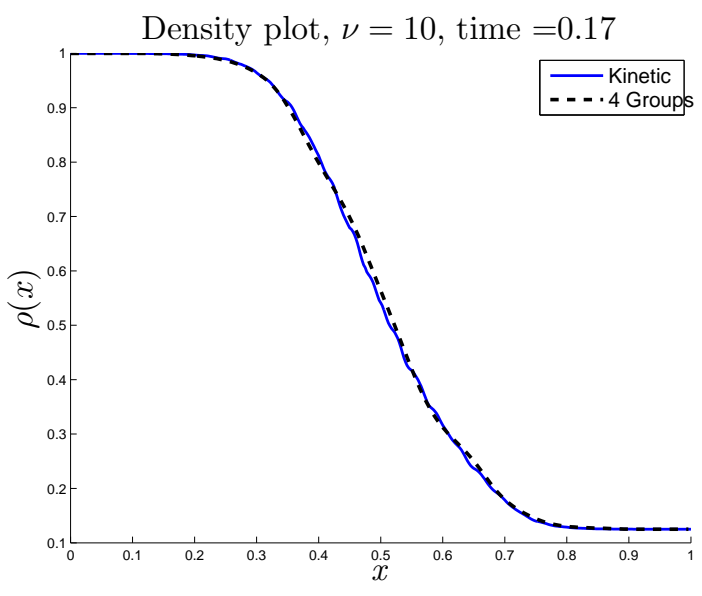

(b) Shock Tube , $\nu=10$ case

Figure 8: Sod Shock Tube Problem Solution, Collision Frequency $v=100,3$ groups (8a), $v=10,4$ groups $(8 b)$

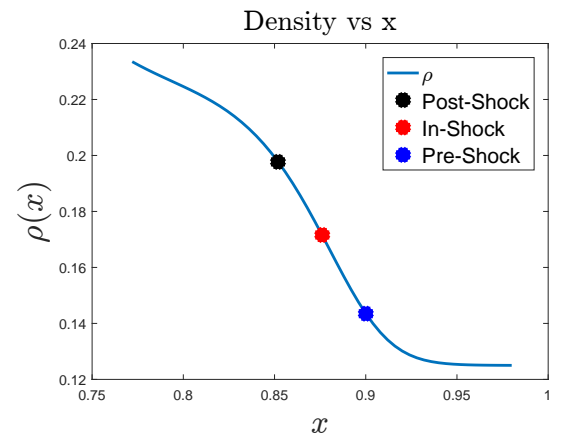

(a) Shock Schematic

\section{Distribution Function around shock location}

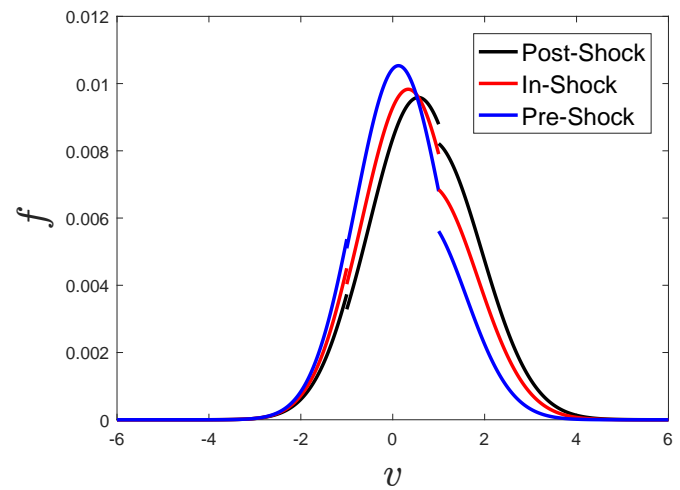

(b) Distribution Function

Figure 9: Distribution Function in the Shock Region, $\nu=100$

Next, the model solution for the case of $\nu=100$ is compared with the corresponding kinetic simulation solution in Figure 8a. A low collision frequency value corresponds fewer molecular collisions and as a result, the shock and the expansion fan appear to be more diffuse than the Euler case. On further reducing the collision frequency to $\nu=10$, we observe that the shock and contact discontinuities observed previously completely vanish. This is shown in (Figure. 8b). For collision frequency of $\nu=10$ and lower, the velocity space needs to be divided into a larger number of groups to accurately capture the macroscopic moments. Here, we use 4 groups for $\nu=10$. 
The distribution function within the shock is plotted $\nu=100$. (Figure 9b). For the low collision frequency case, the effect of non-equilibrium is observed in the distribution function. Discontinuities exist in the distribution function because the model does not enforce continuity of the distribution function at the group boundaries. Using 3 or 4 groups, the macroscopic moments of the non-equilibrium are captured with very good accuracy. The microscopic distribution can be captured if a large number of groups are used for the model.

\section{Conclusion}

In this study, a macroscopic model based on the multi-group maximum entropy principle for studying flows in translational non-equilibrium is developed. The underlying basis of constructing the model and the governing equations is discussed. The model is used for the solution of a zero dimensional relaxation test case and a one dimensional shock tube problem, and the model results are compared with the Boltzmann equation's solution. It is observed that the model is able to capture the macroscopic moments really well over a wide range of collision frequencies.

The success of the model in capturing the physics of simple test cases motivates further development of the model. In this regard, future work for the current model involves the development of better numerical schemes for model solution and proving the hyperbolic and conservation properties of the model. In addition, extension of the model to a more complex collision operator like the Fokker Collision Integral will allow for a more detailed model solution. These studies will be performed in the future.

\section{Appendix}

This section summarizes mathematical expressions for the group density, velocity, energy, and their corresponding fluxes in terms of the group distribution function parameters.

\section{A. Function Form}

The functional form of any group distribution function is given by:

$$
f(x, \vec{c}, t)=A \exp \left(-\beta\left(\left(c_{x}-w\right)^{2}+c_{y}^{2}+c_{z}^{2}\right)\right)
$$

In the above equation, the spatial and temporal variations determined by the parameters $\beta, w$ and $A$.

\section{B. Function Definition - $I_{n x}$}

The following notation will be used for expressing certain functions:

$$
I_{n x}=\int_{c_{x}=c_{i}}^{c_{x}=c_{f}}\left(c_{x}-w\right)^{n} \exp \left(-\beta\left(c_{x}-w\right)^{2}\right) d c_{x}
$$

The expressions that occur in the flux and conserved variables vector are expanded and written as follows:

$$
\begin{gathered}
I_{0 x}=\sqrt{\frac{\pi}{4 \beta}}\left(\operatorname{erf}\left(\sqrt{\beta}\left(c_{f}-w\right)\right)-\operatorname{erf}\left(\sqrt{\beta}\left(c_{i}-w\right)\right)\right) \\
I_{1 x}=\left(\frac{\exp \left(-\beta\left(c_{i}-w\right)^{2}\right)-\exp \left(-\beta\left(c_{f}-w\right)^{2}\right)}{2 \beta}\right) \\
I_{2 x}=-\frac{\sqrt{\pi}}{2 \sqrt{\beta}}\left(\frac{\exp \left(-\beta\left(c_{f}-w\right)^{2}\right)\left(c_{f}-w\right)}{\sqrt{\pi \beta}}-\frac{\exp \left(-\beta\left(c_{i}-w\right)^{2}\right)\left(c_{i}-w\right)}{\sqrt{\pi \beta}}\right) \\
+\left(\frac{\sqrt{\pi}}{4 \sqrt{\beta^{3}}}\right)\left(\operatorname{erf}\left[\sqrt{\beta}\left(c_{f}-w\right)\right]-\operatorname{erf}\left[\sqrt{\beta_{k}}\left(c_{i}-w\right)\right]\right) \\
I_{3 x}=-\left(\frac{\exp \left(-\beta\left(c_{f}-w\right)^{2}\right)\left(c_{f}-w\right)^{2}-\exp \left(-\beta\left(c_{i}-w\right)^{2}\right)\left(c_{i}-w\right)^{2}}{2 \beta}+\frac{\exp \left(-\beta\left(c_{f}-w\right)^{2}\right)-\exp \left(-\beta\left(c_{i}-w\right)^{2}\right)}{2 \beta^{2}}\right)
\end{gathered}
$$




\section{C. $\vec{U}$ and $\vec{F}$ expressions}

The expression for $\vec{U}$ is given by:

$$
\begin{gathered}
\vec{U}=\left[\begin{array}{c}
n A \frac{\pi}{\beta} I_{0 x} \\
n A \frac{\pi}{\beta}\left(I_{1 x}+w I_{0 x}\right) \\
n A \frac{\pi}{\beta}\left(I_{2 x}+w^{2} I_{0 x}+2 w I_{1 x}+\frac{I_{0 x}}{\beta}\right)
\end{array}\right] \\
F=\left[\begin{array}{c}
n A \frac{\pi}{\beta}\left(I_{1 x}+w I_{0 x}\right) \\
n A \frac{\pi}{\beta}\left(I_{2 x}+w^{2} I_{0 x}+2 w I_{1 x}\right) \\
n A \frac{\pi}{\beta}\left(I_{3 x}+I_{0 x} w^{3}+3 I_{1 x} w^{2}+3 I_{2 x} w\right)+\frac{n A \pi\left(I_{1 x}+w I_{0 x}\right)}{\beta^{2}}
\end{array}\right]
\end{gathered}
$$

\section{Acknowledgments}

V.Jayaraman and M.Panesi were supported by the AE Multidisciplinary Initiative, Department of Aerospace Engineering, University of Illinois at Urbana-Champaign.

\section{References}

${ }^{1}$ Bhatnagar, P. L., Gross, E. P., and Krook, M., "A model for collision processes in gases. I. Small amplitude processes in charged and neutral one-component systems," Physical review, Vol. 94, No. 3, 1954, pp. 511.

${ }^{2}$ Gnoffo, P. A., "Planetary-Entry Gas Dynamics 1," Annual Review of Fluid Mechanics, Vol. 31, No. 1, 1999, pp. 459-494.

${ }^{3}$ Vincenti, W. G. and Kruger, C. H., "Introduction to physical gas dynamics," Introduction to physical gas dynamics, by Vincenti, Walter Guido; Kruger, Charles H. New York, Wiley [1965], Vol. 1, 1965.

${ }^{4}$ Chapman, S. and Cowling, T. G., The mathematical theory of non-uniform gases: an account of the kinetic theory of viscosity, thermal conduction and diffusion in gases, Cambridge university press, 1970.

${ }^{5} \mathrm{Grad}, \mathrm{H}$. , "On the kinetic theory of rarefied gases," Communications on pure and applied mathematics, Vol. 2, No. 4, 1949, pp. 331-407.

${ }^{6}$ Levermore, C. D., "Moment closure hierarchies for kinetic theories," Journal of statistical Physics, Vol. 83, No. 5-6, 1996, pp. 1021-1065.

${ }^{7}$ Liu, Y., Panesi, M., Sahai, A., and Vinokur, M., "General multi-group macroscopic modeling for thermo-chemical nonequilibrium gas mixtures," The Journal of chemical physics, Vol. 142, No. 13, 2015, pp. 134109.

${ }^{8}$ Torrilhon, M., "Modeling nonequilibrium gas flow based on moment equations," Annual Review of Fluid Mechanics, Vol. 48, 2016, pp. 429-458.

${ }^{9}$ Liu, Y., Vinokur, M., Panesi, M., and Magin, T., "A multi-group maximum entropy model for thermo-chemical nonequilibrium," AIAA paper, Vol. 4332, 2010, pp. 2010.

${ }^{10}$ Crouseilles, N., Degond, P., and Lemou, M., "A hybrid kinetic/fluid model for solving the gas dynamics Boltzmann-BGK equation," Journal of Computational Physics, Vol. 199, No. 2, 2004, pp. 776-808.

${ }^{11}$ Dubroca, B. and Klar, A., "Prise en compte d'un fort déséquilibre cinétique par un modèle aux demi-moments," Comptes Rendus Mathematique, Vol. 335, No. 8, 2002, pp. 699-704.

${ }^{12}$ Liepmann, H. W., Narasimha, R., and Chahine, M. T., "Structure of a plane shock layer," The Physics of Fluids, Vol. 5, No. 11, 1962, pp. 1313-1324.

${ }^{13}$ Shannon, C. E., "A mathematical theory of communication," ACM SIGMOBILE Mobile Computing and Communications Review, Vol. 5, No. 1, 2001, pp. 3-55.

${ }^{14}$ Jaynes, E. T., "Information theory and statistical mechanics," Physical review, Vol. 106, No. 4, 1957, pp. 620.

${ }^{15}$ Arroyo, M. and Ortiz, M., "Local maximum-entropy approximation schemes: a seamless bridge between finite elements and meshfree methods," International journal for numerical methods in engineering, Vol. 65, No. 13, 2006, pp. $2167-2202$.

${ }^{16}$ Mead, L. R. and Papanicolaou, N., "Maximum entropy in the problem of moments," Journal of Mathematical Physics, Vol. 25, No. 8, 1984, pp. 2404-2417.

${ }^{17} \mathrm{Sod}, \mathrm{G}$. A., "A survey of several finite difference methods for systems of nonlinear hyperbolic conservation laws," Journal of computational physics, Vol. 27, No. 1, 1978, pp. 1-31.

${ }^{18}$ Alekseenko, A. and Josyula, E., "Deterministic solution of the spatially homogeneous Boltzmann equation using discontinuous Galerkin discretizations in the velocity space," Journal of Computational Physics, Vol. 272, 2014, pp. 170-188.

${ }^{19}$ Alekseenko, A., "Numerical properties of high order discrete velocity solutions to the BGK kinetic equation," Applied Numerical Mathematics, Vol. 61, No. 4, 2011, pp. 410-427. 